\title{
ESTUDIO DE ÁNGULOS INSCRIPTOS EN CIRCUNFERENCIAS CON GEOGEBRA $^{\circledR}$
}

\author{
Yésica Muruaga ${ }^{1}$ \\ Escuela de Educación Secundaria $N^{\circ}$ 3. Bragado. Buenos Aires \\ Ana Rosa Corica ${ }^{2}$ \\ Consejo Nacional de Investigaciones Científicas y Técnicas (CONICET) \\ Núcleo de Investigación en Educación en Ciencia y Tecnología (NIECyT) - Facultad de \\ Ciencias Exactas de la Universidad Nacional del Centro de la Provincia de Buenos Aires
}

Recibido: 29/10/2014

Aceptado: 17/04/2015

\section{Resumen:}

En este trabajo se reportan resultados del diseño e implementación de una Actividad de Estudio e Investigación para el estudio de ángulos inscriptos y cuadriláteros cíclicos en la escuela secundaria. Con fundamento en la Teoría Antropológica de lo Didáctico diseñamos un dispositivo didáctico que involucra el estudio de tareas con lápiz y papel y con el empleo del software Geogebra ${ }^{\circledR}$.La combinación de tareas permitió la integración de nociones en el estudio de cuadriláteros cíclicos que se proponen estudiar de manera aislada en el diseño curricular.

Palabras clave: Actividad de estudio e investigación, geometría, enseñanza; geogebra ${ }^{\circledR}$.

\begin{abstract}
:
In this work, we show results of the design and implementation of a Study and Research Activity for the study of inscribed angles and cyclic quadrilateral in high school. The didactic device was designed considering the fundaments of the Anthropological Theory of Didactics. The activity involved paper and pencil tasks and the use of Geogebra ${ }^{\circledR}$ software. The combination of tasks allowed the integration of concepts in the study of cyclic quadrilaterals proposed study in isolation in curriculum design.
\end{abstract}

Keywords: Study and research activity, geometry, teaching, geogebra ${ }^{\circledR}$.

\footnotetext{
1 yesicamuruaga@yahoo.com.ar

2 acorica@exa.unicen.edu.ar
} 


\section{INTRODUCCIÓN}

Diversos estudios indican las dificultades de los estudiantes en la comprensión de nociones geométricas (Andrade, Nacarato, 2004). En particular, la relevancia que tiene demostrar en geometría y las dificultades detectadas en los estudiantes en comprender y elaborar tales demostraciones condujeron a diversos investigadores a elaborar dispositivos didácticos con software de geometría dinámica (Corrales, 2011; García, Arriero, 2000; González, Lupiáñez, 2001; Hoyos, 2006; Iaranzo, Fortuny, 2009; Pérez, 2000; Pichel, 2000; Richard, 2010). El Software para Geometría Dinámica (SGD), es una herramienta que proporciona un medio para la manipulación directa de las representaciones de los objetos geométricos a través de su principal rasgo que es el arrastre. Permite construir significado de los objetos geométricos a través de la posibilidad de transformación continua de los dibujos, y que son diferentes a los construidos al utilizar papel y lápiz (Acosta, 2005, Larios, González, 2009). El dinamismo proporcionado por el SGD permite la exploración de situaciones geométricas permitiendo generalizar situaciones y buscar propiedades invariantes a partir de casos particulares. Esto involucra un trabajo exploratorio que implica realizar ensayos, equivocarse, reajustar, intentos de explicar lo que sucede, establecer si se puede armar o no un dibujo. Así la comprensión y la explicación de las resoluciones demandan del uso de propiedades, y se pone de manifiesto que en geometría ver y dibujar son insuficientes (Itzcovich, 2005). El SGD destaca la diferencia entre dibujo y figura, pues en este ambiente las construcciones geométricas se encuentran realizadas con base en las relaciones lógicas entre los objetos y no solo sobre los aspectos figurales de las mismas, permitiendo que al momento de hacer una construcción por medio del arrastre, las propiedades geométricas se mantengan invariantes (Larios, González, 2009). En este sentido, Marrades y Gutierrez (2000, 119-120) afirman que "el modo de arrastre es una de las características únicas de los SGD que hace entornos mucho más poderosos que el aprendizaje tradicional con lápiz y papel. El modo de arrastre permite a los estudiantes ver tantos ejemplos como sea necesario en unos pocos segundos, y les proporciona un inmediato feedback que no puede ser obtenido en la enseñanza con lápiz y papel”. Esta característica viabiliza trabajar con demostraciones en ambientes 
dinámicos, en los que los estudiantes pueden investigar y conjeturar más de lo que pueden realizar con actividades en lápiz y papel (Paulek, Dias, 2013).

En este trabajo retomamos preguntas que surgen del trabajo de Marín (2012), tal como es: ¿Cómo dar sentido al estudio de ángulos inscriptos en una circunferencia, dado que su estudio se propone de manera aislada en el diseño curricular? La autora diseñó e implementó un dispositivo didáctico para el estudio de ángulos inscriptos en circunferencias con tareas de lápiz y papel. La propuesta permitió involucrar a los estudiantes en un trabajo distanciado de la enseñanza tradicional, que implicó modificaciones a nivel de mesogénesis, topogénesis y cronogénesis.

En este trabajo se retoma la propuesta de Marín (2012). Diseñamos e implementamos una Actividad de Estudio e Investigación para el estudio de ángulos inscriptos en circunferencias y cuadriláteros cíclicos con el empleo de Geogebra ${ }^{\circledR}$. En particular, el estudio de ángulos inscriptos en circunferencias gesta un entorno tecnológico que justifica, en parte, el estudio de las propiedades y relaciones de los cuadriláteros cíclicos. Estas últimas nociones se encuentran excluidas del diseño curricular de la escuela secundaria argentina, y son en ellas donde consideramos que cobra sentido el estudio de ángulos inscriptos en circunferencias.

\section{EL DISEÑO CURRICULAR DE LA ESCUELA SECUNDARIA EN RELACIÓN A LA ENSEÑANZA DE NOCIONES DE GEOMETRÍA}

El Diseño Curricular para la Educación Secundaria de la provincia de Buenos Aires en Argentina (DGCyE, 2008, 2009) propone para el segundo año (estudiantes de 13 - 14 años), dentro del eje de Geometría y Magnitudes, estudiar la noción de Lugar Geométrico, y en particular la Circunferencia. Se propone reconocer ángulos centrales, inscriptos y semi - inscriptos en una circunferencia, explorar y validar sus propiedades.

En el Diseño Curricular para tercer año (estudiantes de 14 - 15 años), en el bloque Geometría y Magnitudes, se propone el estudio de figuras planas. Se sugiere plantear problemas tales que permitan revisar los conocimientos previos de los estudiantes sobre ángulos en la circunferencia. El estudio propuesto no implica abordar tareas que retomen lo estudiado en años anteriores y así modificar las técnicas y tecnologías conocidas y proseguir en la elaboración de nuevas. 
El estudio de ángulos inscriptos en circunferencias se encuentra desarticulado con relación a las restantes nociones que se proponen estudiar. Se sugiere iniciar el estudio en segundo año de la secundaria, y que se retome al año siguiente como revisión.

\section{MARCO TEÓRICO}

En este trabajo adoptamos como referencial teórico a la Teoría Antropológica de lo Didáctico (Chevallard, 1999, 2007, 2009a, 2009b). La noción de praxeología u organización matemática (OM) constituye el constructo fundamental de la teoría. Así, toda actividad humana regularmente realizada se modeliza con la noción de praxeología. Estas emergen como respuesta a una cuestión o conjunto de cuestiones problemáticas que se denominan cuestiones generatrices.

Las praxeologías se componen de dos niveles: el nivel de la praxis o del saber hacer, que engloba un cierto tipo de tareas, así como las técnicas para resolverlos; y el nivel del logos o del saber, en el que se sitúan los discursos que describen, explican y justifican las técnicas que se utilizan, los que reciben el nombre de tecnología. Dentro del saber se postula un segundo nivel de descripción-explicación-justificación que se denomina teoría.

En la TAD las formas de organizar la enseñanza escolar de la matemática, se describe en términos de praxeologías didácticas. En particular, en los diversos procesos de construcción matemática se pueden identificar aspectos invariantes. Así, el proceso de estudio se sitúa en un espacio determinado por seis momentos didácticos: el momento del primer encuentro con un determinado tipo de tareas; el momento exploratorio del tipo de tareas; el momento de construcción de un entorno tecnológicoteórico, que explica y justifica las técnicas puestas en funcionamiento y permite la elaboración de nuevas técnicas; el momento de trabajo de la técnica, que provoca la evolución de las técnicas existentes y la construcción de nuevas; el momento de la institucionalización, que delimita y precisa aquellos elementos que conforman la organización matemática construida; el momento de la evaluación de la praxeología construida.

Los desarrollos de los últimos años de la TAD ponen de manifiesto la necesidad de introducir en los sistemas de enseñanza procesos de estudio funcionales, donde los 
saberes no constituyan monumentos que el profesor enseña a los estudiantes, sino herramientas materiales y conceptuales, útiles para estudiar y resolver situaciones problemáticas. Así, las Actividades de Estudio e Investigación (AEI) emergen como modelo didáctico para abordar dicha problemática. Una enseñanza por AEI implica el estudio de cuestiones ricas, vivas y fecundas que provoquen en los estudiantes la necesidad de seguir aprendiendo, y generar un proceso de investigación, en el que se debe explorar, conjeturar y validar.

\section{METODOLOGÍA}

La metodología propuesta fue de corte cualitativa, y el diseño de investigación es de corte exploratorio y descriptivo (Hernández, Fernández, Baptista, 2010). En este trabajo estudiamos una problemática poco explorada, como es la enseñanza de ángulos inscriptos y cuadriláteros cíclicos en la escuela secundaria. Diseñamos e implementamos una AEI que involucra el estudio de actividades con Geogebra ${ }^{\circledR}$.

Según el referencial teórico adoptado, como actividad previa al diseño de la AEI es necesario elaborar un Modelo Praxeológico de Referencia (MPR). Este modelo se encuentra estrechamente relacionado con lo que se entiende por enseñar y aprender matemática en una cierta institución. Para el diseño de la AEI trabajamos sobre el MPR propuesto por Corica y Marín (2014) y Marín (2012).

\subsection{Descripción de las características del curso seleccionado}

La implementación se desarrolló en un curso de segundo año de una escuela pública agraria en Argentina. La escuela se caracteriza por tener un sistema denominado de alternancia. Los alumnos permanecen una semana en la institución y dos semanas en sus hogares. La implementación de la AEI tuvo lugar luego de 2 meses de iniciado el ciclo lectivo, por uno de los profesores investigadores, durante 8 encuentros de 120 minutos de duración cada uno. La comunidad de estudio estaba constituida por $\mathrm{N}=12$ alumnos, que formaron pequeños grupos de trabajo compuestos por 3 alumnos.

$\mathrm{Al}$ momento de implementar, en el curso predominaba una enseñanza tradicional. El protagonista del proceso de estudio era el profesor: es quien proponía las tareas, las técnicas y las validaba durante la implementación de la AEI, se trató de instalar una 
dinámica de estudio en correspondencia con el marco teórico adoptado. Las clases se focalizaron en que los estudiantes propongan las técnicas para resolver las situaciones y que justifiquen sus producciones. Luego de cada resolución el profesor proponía compartir con toda la comunidad de estudio las resoluciones aportadas por cada grupo, lo que permitió confrontar las distintas resoluciones y evaluar las técnicas construidas. Los debates permitieron realizar una síntesis de lo aportado por los alumnos y así institucionalizar los nuevos saberes reconstruidos.

Como el curso tenía un sistema de alternancia y los encuentros se producían cada 15 días, al inicio de cada clase el profesor tuvo que indagar a los estudiantes con el propósito de recuperar las técnicas y tecnologías estudiadas las clases anteriores para proseguir con las sucesivas situaciones planificadas.

\subsection{Recolección de registros}

Se obtuvieron los protocolos escritos de cada uno de los estudiantes, y se tomaron registros de audio general de todas las sesiones. Los protocolos escritos de los estudiantes se retiraron al finalizar cada sesión, se escanearon y se devolvieron en la sesión inmediata siguiente, para garantizar la continuidad de su trabajo y para que los estudiantes dispongan permanentemente de sus registros. También se retiró de cada sesión, los protocolos realizado en Geogebra ${ }^{\circledR}$ por los alumnos.

\section{DISEÑO E IMPLEMENTACIÓN DE LA ACTIVIDAD DE ESTUDIO E INVESTIGACIÓN}

La AEI permite la reconstrucción de una OM cuyo estudio implica vivir todos los momentos de estudio. La AEI se gestó a partir de la cuestión generatriz: $Q_{0}$ : ¿Cuáles son las relaciones que se establecen entre los diferentes ángulos que se tracen en una circunferencia y otros elementos de la misma?

El estudio de $Q_{0}$ conduce a la formulación de cuestiones derivaras $\left(q_{i}\right)$, que involucran el estudio de tipo de tareas $\left(T_{i}\right)$. A partir de estos tipos de tareas establecimos las situaciones que realizaron los estudiantes. El estudio de dichas tareas proporciona una respuesta $\left(R_{i}\right)$ que en conjunto, contribuyen a la elaboración de la respuesta $R$ a $Q_{0}$.En el siguiente esquema presentamos un conjunto reducido de cuestiones derivadas 
de $Q_{0}$, que se corresponden con los objetivos del trabajo y las conexiones entre las respuestas $R_{i}$ que emergen del estudio de cada $T_{i}$.

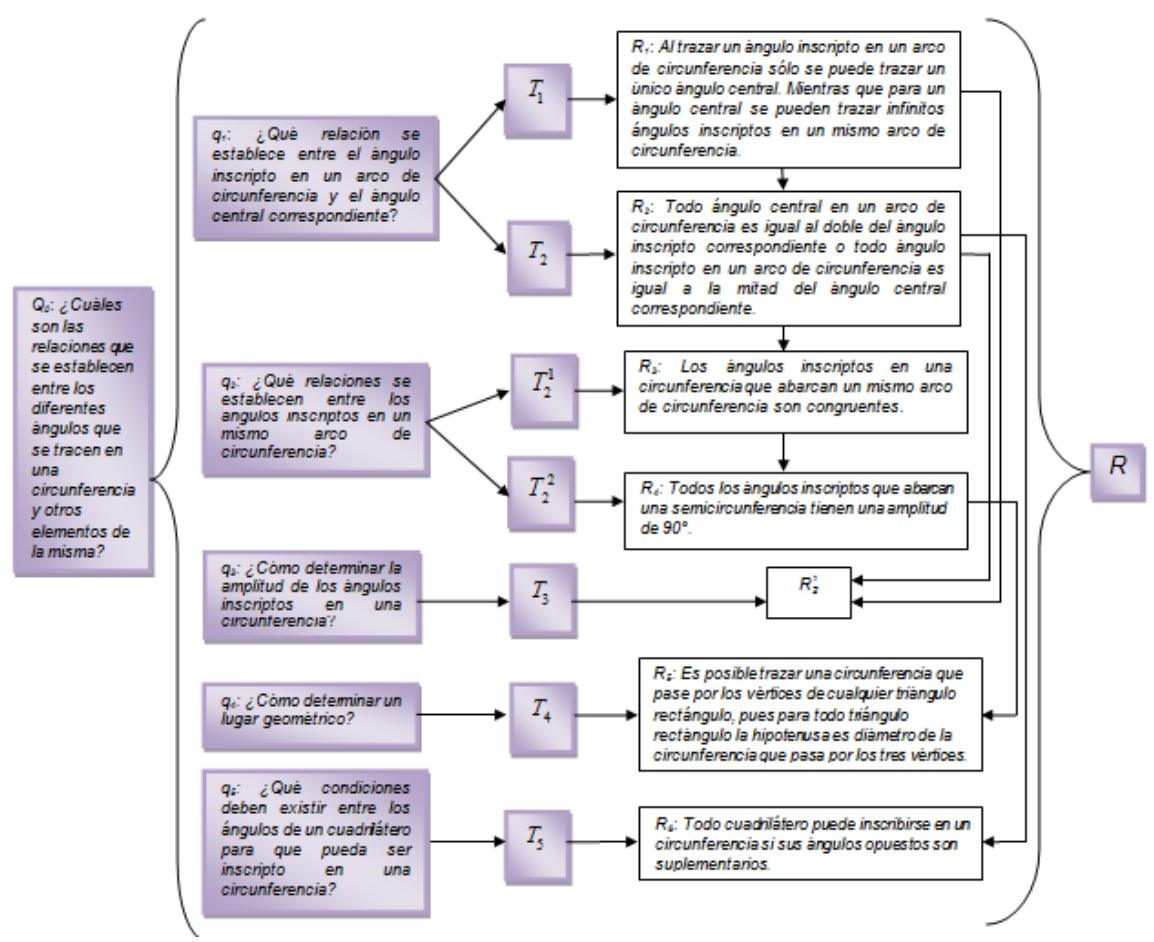

Figura 1. Esquema de la AEI

De la cuestión $Q_{0}$ derivamos cinco cuestiones fundamentales. La primera cuestión que formulamos es $q_{1}$ : ¿Qué relación se establece entre el ángulo inscripto en un arco de circunferencia y el ángulo central correspondiente?

La cuestión $q_{1}$ conduce al estudio del tipo de tareas $T_{1}$ : Determinar ángulos inscriptos y ángulos centrales en un mismo arco de circunferencia. El estudio de $T_{1}$ conduce a la elaboración de la respuesta $R_{1}$ : Al trazar un ángulo inscripto en un arco de circunferencia sólo se puede trazar un único ángulo central. Mientras que para un ángulo central se pueden trazar infinitos ángulos inscriptos en un mismo arco de circunferencia.

La cuestión $q_{1}$ conduce también al estudio del tipo de tareas $T_{2}$ : Establecer la relación que existe entre los ángulos inscriptos y los ángulos centrales en un mismo arco de circunferencia. El estudio de $T_{2}$ permite elaborar la respuesta $R_{2}$ : Todo ángulo central en un arco de circunferencia es igual al doble del ángulo inscripto 
correspondiente o todo ángulo inscripto en un arco de circunferencia es igual a la mitad del ángulo central correspondiente. Aquí se establece la relación entre un ángulo inscripto y un ángulo central en un mismo arco de circunferencia considerando las conclusiones obtenidas en $R_{1}$ y otras relaciones y propiedades estudiadas por los alumnos en años anteriores. Esta respuesta constituye un teorema fundamental de la OM, pues es la tecnología que justifica parte del hacer de las tareas que se indican a continuación.

La segunda cuestión que proponemos es $q_{2}$ : ¿Qué relaciones se establecen entre los ángulos inscriptos en un mismo arco de circunferencia? Esta cuestión conduce al estudio de dos tipos de tareas, vinculados a $T_{2}: T_{2}^{1}$ : Determinar la relación entre ángulos inscriptos en un mismo arco de circunferencia; y $T_{2}^{2}$ : Determinar la amplitud de los ángulos inscriptos en una semicircunferencia. Con estos tipos de tareas se pretende poner a prueba la potencia de las técnicas que se institucionalizan a partir del estudio de las tareas anteriores.

El estudio de $T_{2}^{1}$ permite elaborar la respuesta $R_{3}$ : Los ángulos inscriptos en una circunferencia que abarcan un mismo arco de circunferencia son congruentes. Las respuestas $R_{2}$ y $R_{3}$ aportan tecnologías para estudiar $T_{2}^{2}$ y elaborar $R_{4}$ : Todos los ángulos inscriptos que abarcan una semicircunferencia tienen una amplitud de $90^{\circ}$.

La tercera cuestión que proponemos es $q_{3}$ : ¿Cómo determinar la amplitud de los ángulos inscriptos en una circunferencia? Esta cuestión conduce al estudio de $T_{3}$ : Determinar la amplitud de ángulos inscriptos en una circunferencia. El estudio de este tipo de tareas requiere del entorno tecnológico gestado en el estudio de $T_{1}$. La elaboración de la respuesta a $q_{3}\left(R_{2}^{1}\right.$,) no aporta nuevos elementos tecnológicos. Para su obtención se requiere del empleo del entorno tecnológico gestado en el estudio de $T_{2}$, y trabajar sobre la técnica producida en $R_{2}$. Así se logra una rutinización de dicha técnica. De esta manera, se obtiene una respuesta $R_{2}^{1}$ que utiliza el entorno tecnológico de $R_{2}$.

La cuarta cuestión es $q_{4}$ : ¿Cómo determinar un lugar geométrico? La cuestión conduce al estudio de $T_{4}$ : Definir un lugar geométrico. Para elaborar una respuesta se requiere del empleo de técnicas institucionalizadas en el estudio de las tareas anteriores. Así, se obtiene una respuesta $R_{5}$ : Es posible trazar una circunferencia que pase por los 
vértices de cualquier triángulo rectángulo, pues para todo triángulo rectángulo la hipotenusa es diámetro de la circunferencia que pasa por los tres vértices.

Finalmente, la quinta cuestión es $q_{5}:$ ¿Qué condiciones deben existir entre los ángulos de un cuadrilátero para que pueda ser inscripto en una circunferencia? Esta cuestión conduce al estudio de $T_{5}$ : Determinar las condiciones para que un cuadrilátero pueda ser inscripto en una circunferencia. Para el hacer de $T_{5}$ se requiere de elementos tecnológicos que emergen de $T_{2}$. Así se obtiene la respuesta $R_{6}$ : Todo cuadrilátero puede inscribirse en una circunferencia si sus ángulos opuestos son suplementarios.

A continuación presentamos 11 situaciones, que derivamos de los tipos de tareas descriptos, y los principales resultados de su implementación.

\subsection{Situación 1}

La situación se corresponde con $T_{1}, T_{2}$, $T_{2}^{1}$. El estudio se inicia identificando ángulos centrales, inscriptos, semiinscriptos, interior y exterior en una circunferencia, utilizando las herramientas proporcionadas por Geogebra ${ }^{\circledR}$.

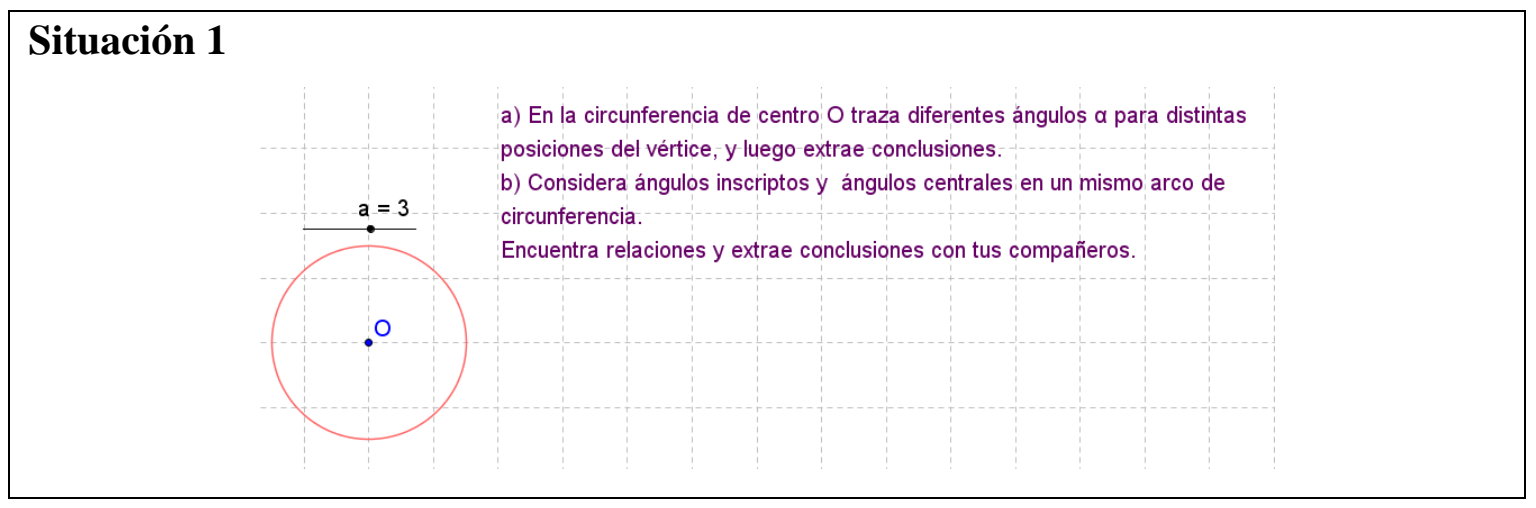

En el estudio de esta situación, el momento prioritario lo constituyó del primer encuentro. En principio, los estudiantes manifestaron dificultades para trazar los ángulos inscriptos correspondientes a cada ángulo central. Esto requirió que el profesor tuviera que sugerir textos para que los alumnos busquen información sobre los ángulos de interés y orientarlos en la realización de las construcciones.

En particular, la experiencia de la construcción, las decisiones del uso de herramientas que brindó el programa, la toma de decisión acerca de la ubicación de las distintas posiciones del vértice y los puntos por los cuales deben pasar los lados de los 
ángulos involucró a los alumnos en un razonamiento con rasgos deductivos. Los estudiantes identificaron ángulos inscriptos y centrales en un mismo arco de circunferencia, y luego establecieron relaciones entre ellos. Si bien se analizaron relaciones entre los ángulos inscriptos y los ángulos centrales en un mismo arco de circunferencia de manera empírica se preparó el acceso de los alumnos a producciones más argumentativas, y todo el proceso deductivo que implican las situaciones que se proponen a continuación.

Luego de las discusiones grupales se acordaron las definiciones que emergieron en la comunidad de estudio. De esta manera, el momento de la institucionalización no quedó exclusivamente en manos del profesor, arribando a las siguientes conclusiones:

En el mismo arco de circunferencia se puede trazar un solo ángulo central con respecto al inscripto trazado. Mientras que en el mismo arco de circunferencia se puede trazar infinitos ángulos inscriptos con respecto al central trazado.

Todos los ángulos inscriptos en un mismo arco de circunferencia son congruentes.

La situación 1.b) tuvo como propósito reconstruir el teorema que establece la relación entre un ángulo inscripto en un arco de circunferencia y el ángulo central correspondiente. Los alumnos debieron establecer la relación entre ángulos, considerando las distintas posiciones del Centro $\mathrm{O}$ respecto al ángulo inscripto, y analizadas en la situación 1 a). En particular, se pretendió que los alumnos recuperaran propiedades conocidas y a partir de ellas elaboraran nuevas.

En particular, para el hacer de la situación 1 b) los integrantes del Grupo 2, realizaron una construcción en lápiz y papel: 


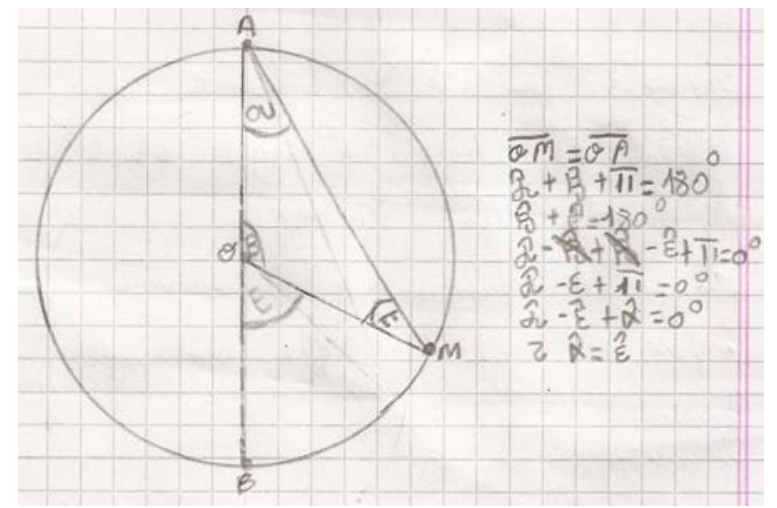

Figura 2. Técnicas propuestas por el Grupo 2 para la situación 1.b)

Los alumnos aplicaron relaciones y propiedades entre los diferentes ángulos del esquema, justificando los procedimientos empleados. En particular, en la resolución de las tareas las justificaciones de los alumnos no se establecieron empíricamente sino que se apoyaron en propiedades y relaciones de los objetos geométricos.

Así, el estudio de la situación 1 permitió concluir en el siguiente teorema:

Todo ángulo central en un arco de circunferencia es igual al doble del ángulo inscripto correspondiente o todo ángulo inscripto en un arco de circunferencia es igual a la mitad del ángulo central correspondiente

\subsection{Situación 2}

La situación 2 se origina a partir de $T_{3}$. El objetivo de la situación fue hacer vivir el momento del trabajo de la técnica. Se inició un trabajo específico sobre las técnicas institucionalizadas para lograr su respectiva rutinización y posterior naturalización.

\section{Situación 2}

Halla la amplitud de $\alpha_{2}$ en cada caso. Indica los procedimientos utilizados para hallar la solución.

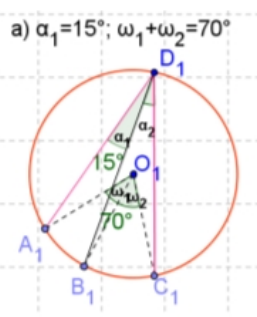

Esquema 1

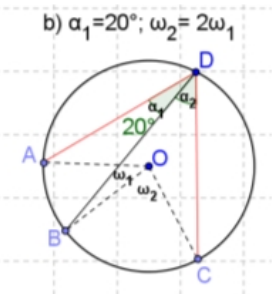

Esquema 2 
En el hacer de los estudiantes para 2 a) el profesor tuvo la necesidad de realizar cuestiones con el fin de que los estudiantes justifiquen las técnicas utilizadas.

En las producciones de los estudiantes para 2 b) se comenzaron a observar mayores vestigios de explicitación del medio tecnológico empleado, tal como ocurre en el siguiente protocolo:

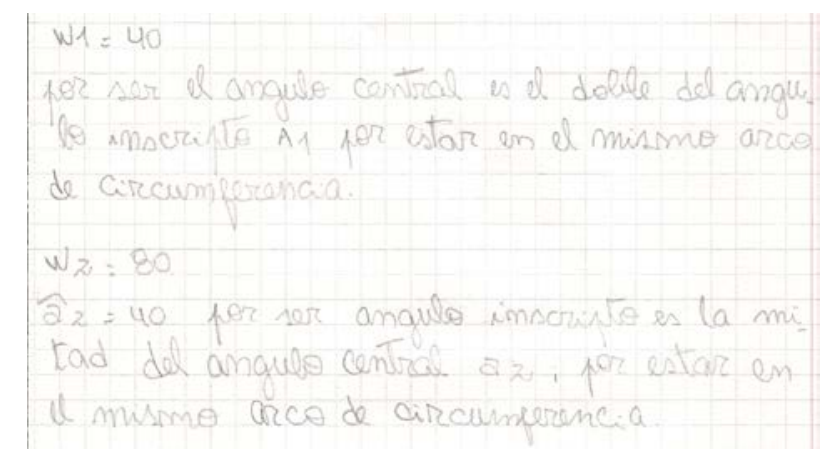

Figura 3. Resolución del Grupo 4 para la tarea 2 b)

\subsection{Situación 3}

La situación 3 se corresponde con $T_{3}$. Se propuso una tarea de lápiz y papel, en la que se indican valores de algunos ángulos en cuadriláteros inscriptos en circunferencias.

\section{Situación 3}

Hallar las medidas de los ángulos marcados en cada figura. Indicar los procedimientos empleados para hallar la solución.
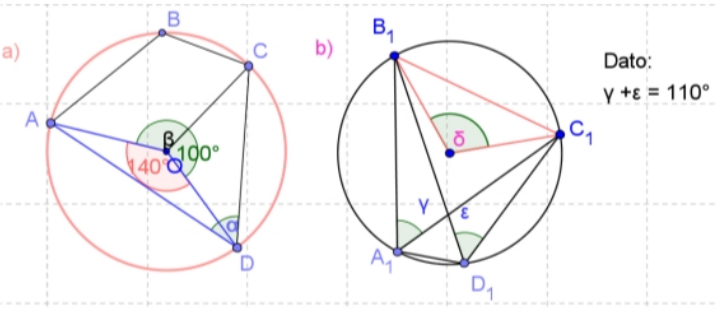
Como se puede observar en el protocolo de la figura 4, los estudiantes utilizaron elementos tecnológicos explícitos que fundamentan la respuesta. El resto de los grupos coincidieron con las relaciones y propiedades utilizadas por este grupo:

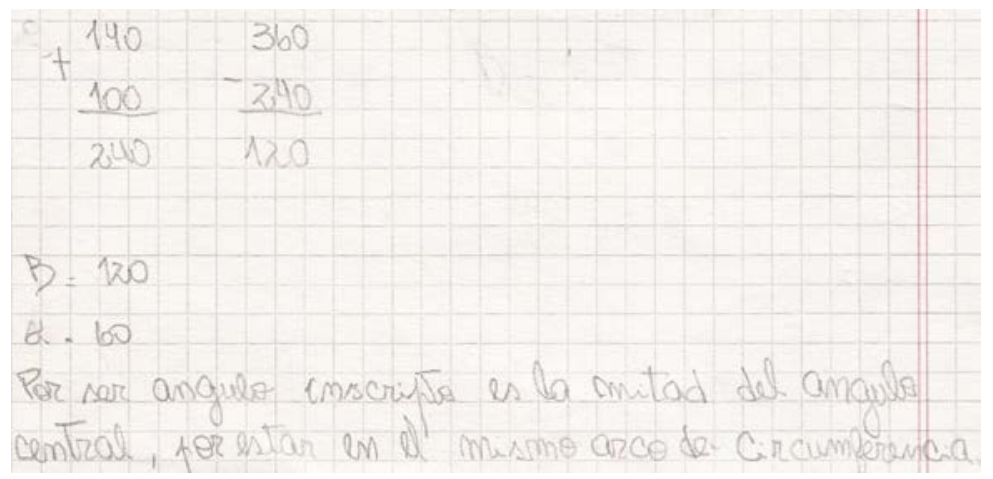

Figura 4. Resolución del Grupo 4 para la tarea 3 a)

Del mismo modo, para el inciso b) las respuestas registradas fueron satisfactorias.

\subsection{Situación 4}

La situación 4 corresponde al tipo de tareas $T_{2}$. La situación se planteó en forma coloquial, y se pretendió que los alumnos realicen sus propios esquemas y encuentren relaciones entre los elementos involucrados. Se trata de una tarea inversa en relación a las propuestas en la situación 2 y 3, y permite que los alumnos empleen el entorno tecnológico gestado en la situación 1.

\section{Situación 4}

a) Construye una circunferencia de centro $O$ y radio 3. Traza un diámetro de extremos A y B. Siendo M un punto perteneciente a la circunferencia, distintos de los extremos A y B. Traza el ángulo BÔM que

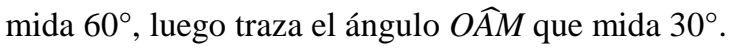

b) Construye una circunferencia de centro $R$ y radio 3. Traza un diámetro de extremos $C$ y D. Siendo $\mathrm{S}$ un punto perteneciente a la circunferencia, distinto de los extremos C y D. Traza el ángulo D $\widehat{R S}$ que mida $100^{\circ}$, luego traza el ángulo $R \widehat{C} S$ que mida $40^{\circ}$.

c) A partir de los resultados obtenidos en a) y b) extrae conclusiones.

En la situación 4, el empleo del software Geogebra ${ }^{\circledR}$ en comparación con la tecnología del papel y el lápiz permitió mantener las relaciones geométricas entre los 
elementos de la construcción. Esto facilitó a los estudiantes, inmersos en el momento exploratorio, concluir en qué situación era posible realizar la construcción y en cuál no. En particular destacamos la siguiente respuesta aportada por el Grupo 4:

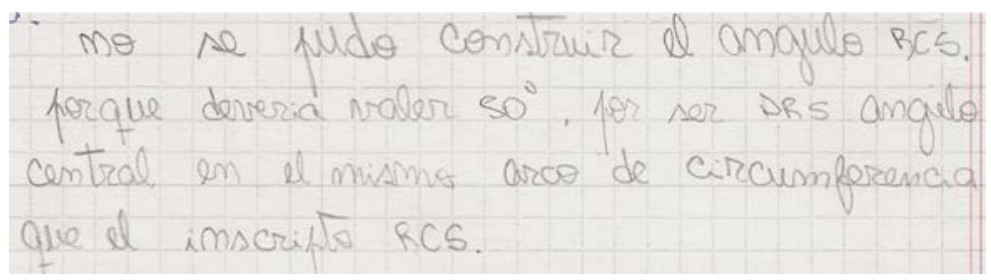

Figura 5. Resolución del Grupo 4 para la tarea 4 b)

Los estudiantes utilizaron un medio tecnológico explícito para justificar las técnicas empleadas. Los demás grupos propusieron respuestas carentes de dicho entorno.

\subsection{Situación 5}

La situación 5 se originó a partir de la tarea $T_{2}^{1}$. El momento didáctico prioritario que se vivió con el estudio de la tarea fue el del trabajo de la técnica.

\section{Situación 5}

Se quiere iluminar con un foco un escenario que ocupa una porción de un predio circular. ¿Cuál es la amplitud del ángulo $\alpha$ que forman los rayos de luz en cada uno de los siguientes escenarios?

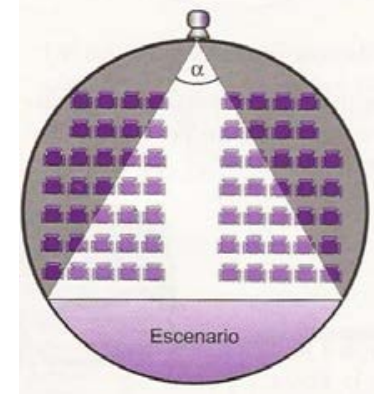

Esquema 5

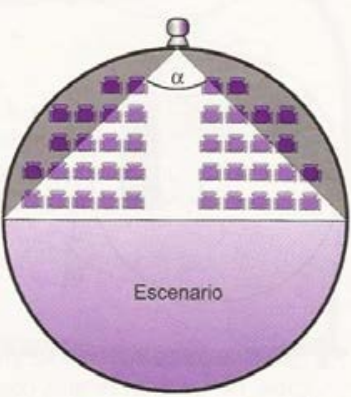

Esquema 6

¿En cuántos lugares diferentes se puede ubicar el foco para que, con el mismo ángulo $\alpha$, se ilumine cada escenario? Fundamenta tu respuesta.

En general, los estudiantes comenzaron el trabajo construyendo el esquema en Geogebra $^{\circledR}$. El empleo del software permitió que los alumnos construyeran esquemas identificando datos e incógnitas, reconociendo características y propiedades de los 
objetos geométricos. El modo de arrastre del programa permitió a los alumnos recuperaran la relación que emergió del estudio de la situación 1 y dar respuesta a la situación 5.

\subsection{Situación 6}

La situación 6 se corresponde con $T_{2}^{2}$. El objetivo de la situación fue que los alumnos puedan determinar la amplitud de los ángulos inscriptos en una semicircunferencia utilizando elementos tecnológicos que emergió del hacer de la situación 1.

\section{Situación 6}

Con el software Geogebra ${ }^{\circledR}$ construye una circunferencia de centro $O$. Marca un segmento $\overline{A B}$ en la circunferencia, y luego determina el ángulo $A \widehat{B} C$ de la medida que elijas.

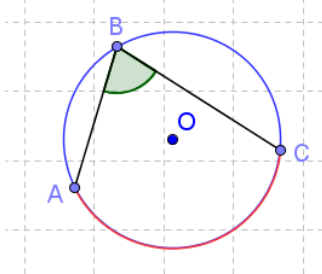

\section{Esquema 7}

a) Mueve el punto B por el arco $\widehat{A C} "$ rojo. Analiza qué sucede con su medida.

b) Mueve el punto $B$ por el arco azul. Analiza qué sucede con la medida del ángulo $A \widehat{B C}$, y qué relación encuentras entre las dos medidas.

c) Determina cuánto mide $A \widehat{B} C$ cuando A y C coinciden con los extremos de un diámetro. Extrae conclusiones de tu propuesta.

Los grupos comenzaron el estudio construyendo la circunferencia en Geogebra ${ }^{\circledR}$ y trazando el ángulo inscripto, como observamos en la producción del Grupo 3:

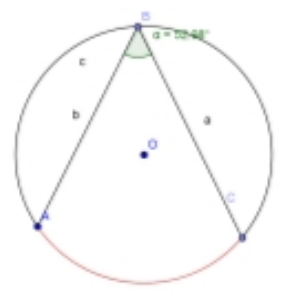

Figura 6. Construcción del Grupo 3 para la situación 6 
Finalizada la construcción, el profesor indicó realizar las tareas a) y b). Aquí se obtuvieron respuestas como la siguiente:

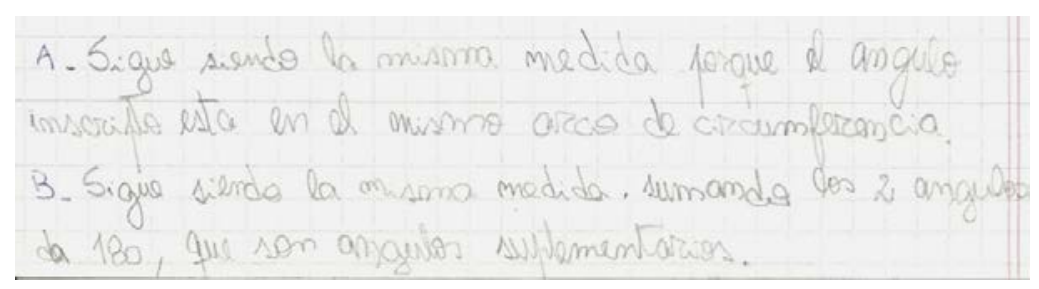

Figura 7. Resolución del Grupo 4 para la situación 6 a) y b)

Los estudiantes decidieron sobre la construcción en Geogebra ${ }^{\circledR}$, ubicando los vértices y los puntos por los que deben pasar los lados de los ángulos inscriptos. Este procedimiento involucra razonamientos con tintes deductivos y promueve la reflexión sobre las condiciones que deben cumplir los ángulos inscriptos en un mismo arco de circunferencia.

En el inciso 6 c), los estudiantes comenzaron el trabajo construyendo el esquema en Geogebra ${ }^{\circledR}$, como se puede observar en la figura 8:

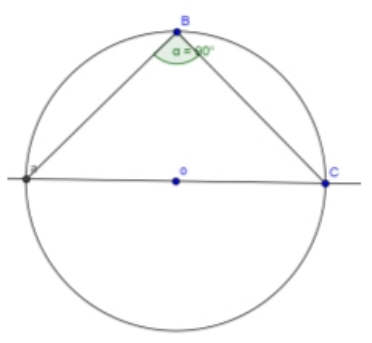

Figura 8. Resolución del Grupo 2 para la tarea 6 c)

En las producciones de los estudiantes sólo el Grupo 4 explicitó el entorno tecnológico que argumenta los procedimientos, tal como se indica en el siguiente protocolo:

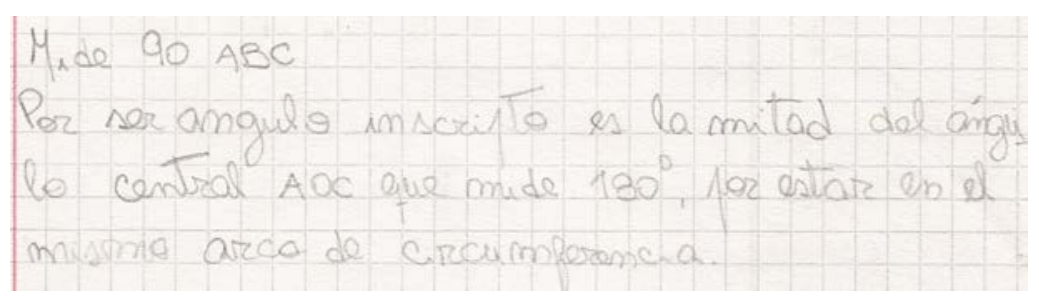

Figura 9. Resolución del Grupo 4 para la tarea 6 c) 
El resto de los grupos explicitaron de manera incompleta el entorno tecnológico. Esto requirió de intervenciones del docente para arribar a una respuesta. Finalmente, en un trabajo conjunto entre los alumnos y el profesor se concluyó en:

Todos los ángulos inscriptos que abarcan una semicircunferencia tienen una amplitud de $90^{\circ}$

\subsection{Situación 7}

La situación 7 corresponde a $T_{2}^{2}$. El estudio de la situación requiere del empleo del entorno tecnológico que emergió del hacer de la situación 1 y 6.

\section{Situación 7}

A, B, C, D y E son puntos de la circunferencia de centro O. Si el ángulo $B \widehat{D C}$ mide $34^{\circ}$, determina la medida de cada uno de los siguientes ángulos: $C \widehat{O B}, D \widehat{E} C, C \widehat{A B}$ y $D \widehat{C} B$. Justifica.

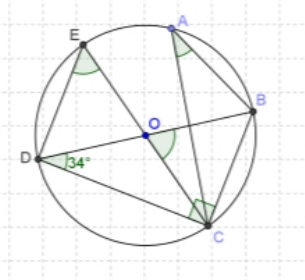

\section{Esquema 8}

En el hacer de los estudiantes, el profesor observó que algunos grupos tenían

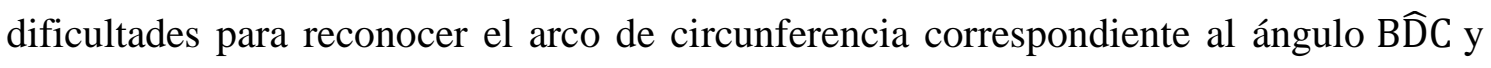
por lo tanto no podían reconocer que los ángulos CÂB y CÔB estaban en el mismo arco de circunferencia que el ángulo BD̂C. Esto requirió que el profesor deba intervenir para lograr que los estudiantes reconozcan los elementos vitales que permiten resolver la tarea.

Así, el Grupo 4 indicó que el ángulo CÂB estaba en el mismo arco de

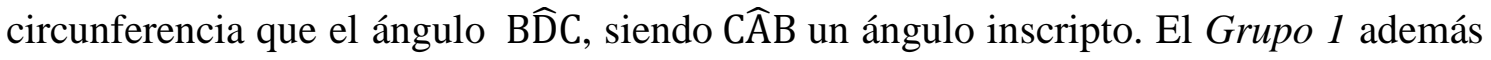
indicó que el ángulo CÔB estaba en el mismo arco de circunferencia que el ángulo BD̂C, siendo el ángulo CÔB un ángulo central. Los demás grupos coincidieron con las respuestas aportadas por el Grupo 1 y Grupo 4.

En particular, se destaca que sólo los Grupos 4 y 2 utilizaron un entorno tecnológico-teórico explícito para argumentar los procedimientos realizados, como observamos en el siguiente protocolo: 


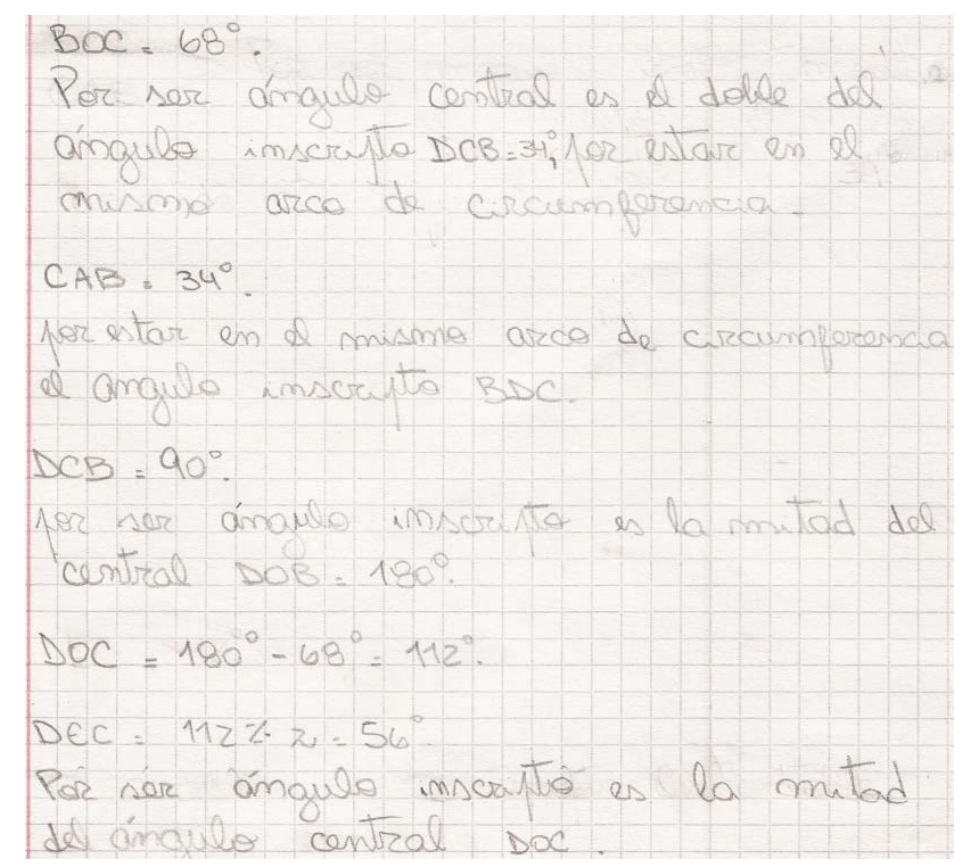

Figura 10. Resolución del Grupo 4 para la tarea 7

Los estudiantes continuaron con la situación 7, para obtener el valor del ángulo inscripto $D \hat{C} B$. Se observó que los grupos recuperaron elementos tecnológicos-teóricos que emergieron del hacer de la situación 6 c).

A continuación, para concluir con la situación 7, el profesor solicitó a los estudiantes indicar la amplitud del ángulo $D \widehat{E} C$. Los estudiantes presentaron dificultades para poder determinar la amplitud de este ángulo, por lo que el profesor tuvo que intervenir. El profesor sugirió que buscaran relaciones entre el ángulo $D \hat{O} C \mathrm{y}$ el ángulo $C \hat{O} B$, pues $D \hat{O} C+C \hat{O} B=180^{\circ}$. Los diferentes grupos consideraron los valores de los ángulos conocidos y establecieron relaciones de acuerdo a los ángulos sugeridos por el docente.

\subsection{Situación 8}

La situación se corresponde con $T_{2}^{2}$. El objetivo fue que los alumnos puedan determinar el centro de una circunferencia a partir de los ángulos inscriptos conocidos.

\section{Situación 8}

¿De qué manera pueden utilizar una escuadra de carpintero para determinar el centro de un círculo? 


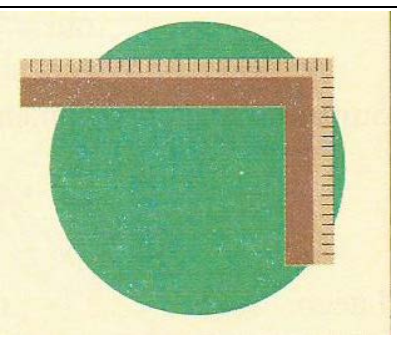

Esquema 9

La situación 8 responde a una situación abierta en el sentido de que los alumnos tuvieron que determinar cuáles son las incógnitas y los datos.

Destacamos la iniciativa del Grupo 1 que propuso resolver mediante el empleo de Geogebra ${ }^{\circledR}$, para poder determinar el centro de la circunferencia y observar de qué manera podían utilizar la escuadra. Los estudiantes explicaron su propuesta del siguiente modo:

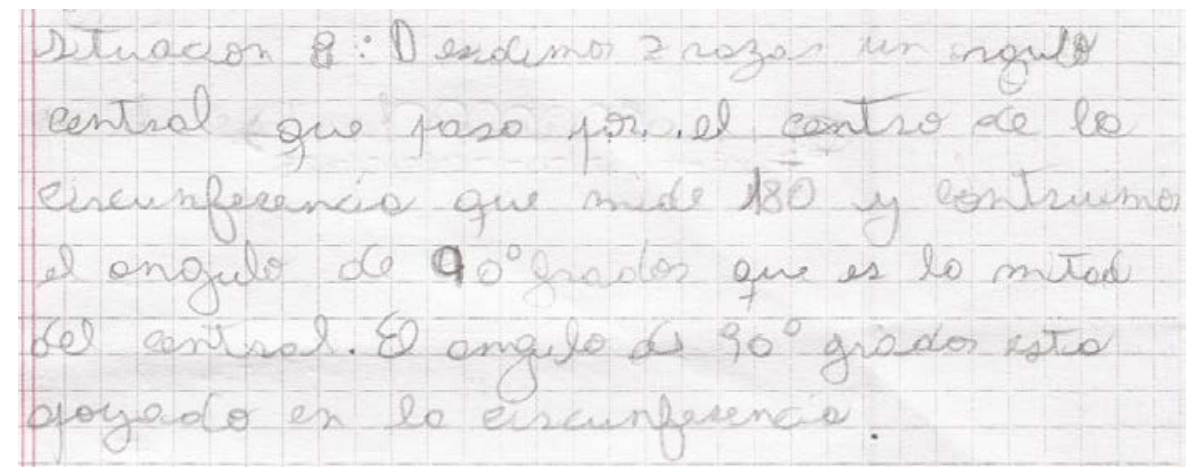

Figura 11. Resolución del Grupo 1 para la tarea 8.

Los alumnos analizaron los datos con los que se debía construir la figura, determinaron si la construcción era posible o no, establecieron relaciones entre los datos conocidos y el dibujo a obtener, sin embargo el entorno tecnológico que se explicitaron fue incompleto. Los estudiantes del Grupo 4 aportaron una respuesta explicitando el entorno tecnológico que justifica las técnicas empleadas: los ángulos inscriptos que miden $90^{\circ}$, coinciden con el diámetro de la circunferencia, permitiendo así encontrar el centro de la misma. 


\subsection{Situación 9}

La situación 9 se corresponde con $T_{5}$. Con esta situación se sumergió a los estudiantes en el momento exploratorio, con el objeto de analizar las condiciones para que un cuadrilátero resulte inscripto en una circunferencia.

\section{Situación 9}

Dibuja en Geogebra ${ }^{\circledR}$ diversas circunferencias e inscríbele cuadriláteros. ¿Cuáles son las condiciones para que sean inscriptibles?

En el hacer de la tarea se observó que solo los estudiantes del Grupo 3 pudieron aportar una respuesta, pues indicaron:

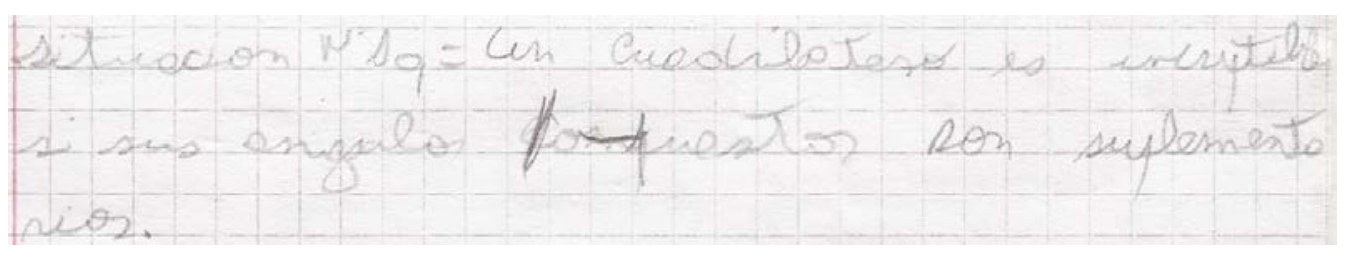

Figura 12. Resolución del Grupo 3 para la tarea 9.

Como el resto de los grupos no aportaron respuesta, el profesor solicitó que demostraran lo indicado por el Grupo 3.

Los estudiantes realizaron construcciones con Geogebra ${ }^{\circledR}$ y luego de una discusión grupal, concluyeron en que un cuadrilátero puede ser inscripto en una circunferencia si los ángulos opuestos son suplementarios. Así, el Grupo 4 indicó:

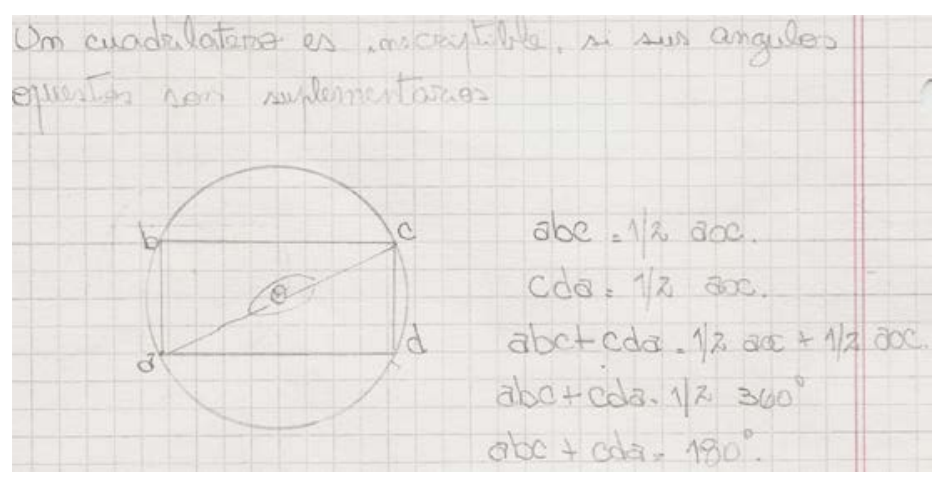

Figura 13. Resolución del Grupo 4 para la demostración de la situación 9. 
En el protocolo observamos que los estudiantes explicitaron elementos tecnológicos para fundamentar la respuesta. Los estudiantes emplearon las técnicas estudiadas y provocaron la aparición de una nueva tecnología. Luego de discusiones grupales se acordó en lo siguiente:

Un cuadrilátero es inscriptible si sus ángulos opuestos son suplementarios.

Con el propósito de profundizar en las condiciones para inscribir cuadriláteros, el profesor propuso la realización de las siguientes tareas con Geogebra ${ }^{\circledR}$ :

a) ¿Es posible trazar la circunferencia que pasa por los vértices de un trapecio? ¿Por qué?

b) ¿Todo rombo puede ser inscripto en una circunferencia? ¿Y el romboide? ¿Por qué?

c) ¿Puede inscribirse un paralelogramo en una circunferencia? ¿Por qué?

Aquí observamos que los estudiantes no propusieron otras resoluciones más que lo aportado por el software. En esta instancia, como el profesor no continuó indagando, no emergieron producciones donde se demuestre lo que el software permite visualizar. Es decir, para los estudiantes las resoluciones experimentales son suficientes para asegurar las características de las figuras.

\subsection{Situación 10}

La situación se corresponde con $T_{5}$. El hacer de la misma involucra elementos tecnológicos institucionalizados en tareas anteriores.

\section{Situación 10}

¿Es posible construir un cuadrilátero cuyas diagonales sean diámetros de la circunferencia?¿Por qué?

Los distintos grupos dibujaron la circunferencia con lápiz y papel. El Grupo 4 propuso la siguiente construcción: 


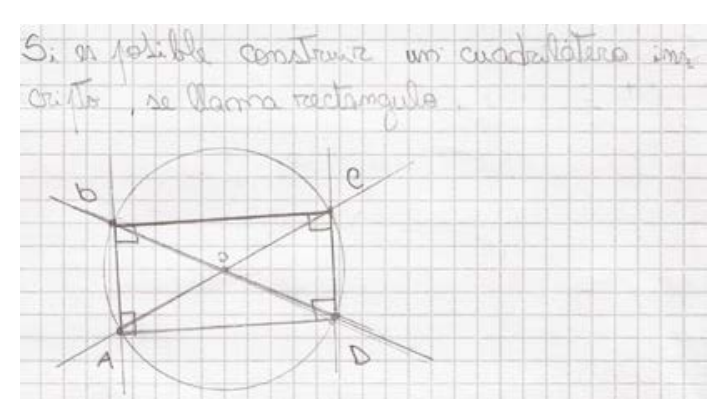

Figura 14. Esquema del Grupo 4 para la situación 10.

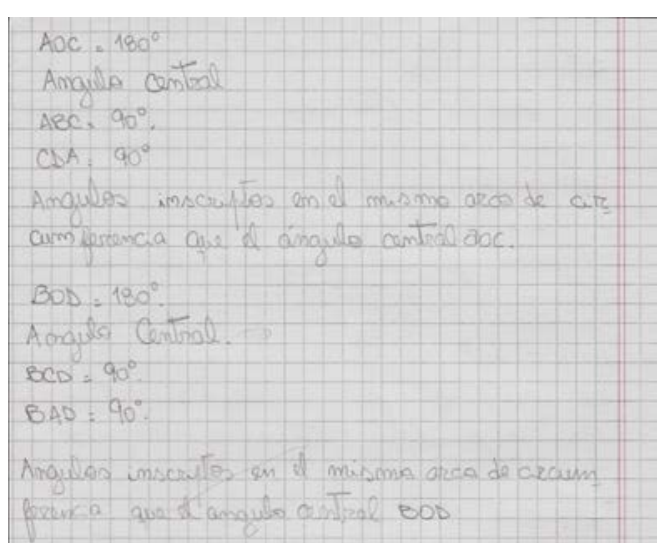

Figura 15. Resolución del Grupo 4 para

la demostración de la situación 10.

Los estudiantes explicitaron elementos tecnológicos que fundamentan la respuesta. El resto de los grupos coincidieron con la propuesta del Grupo 4. Recuperaron propiedades estudiadas años anteriores y pusieron en práctica técnicas provenientes de la situación 1. Esto permitió trabajar con argumentos deductivos, llegando al resultado de manera independiente a la experimentación. Finalmente, la comunidad concluyó en lo siguiente:

Solo los rectángulos inscriptos tienen como diagonales los diámetros de la circunferencia.

\subsection{Situación 11}

La situación 11 se corresponde con el tipo de tareas T5. El objetivo fue que los alumnos puedan determinar la amplitud de los ángulos inscriptos en una circunferencia utilizando elementos tecnológicos que emergen del hacer de las situaciones 1 y 9.

\section{Situación 11}

Calcula los ángulos interiores del trapecio isósceles inscripto.

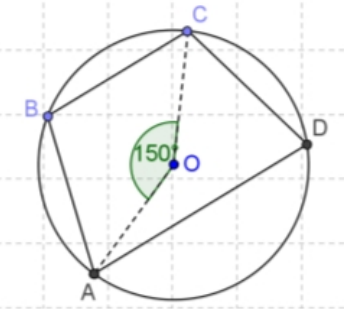

Esquema 10 
En los protocolos de los estudiantes observamos explícito el medio tecnológico que justifica las técnicas propuestas, tal como se puede observar a continuación:

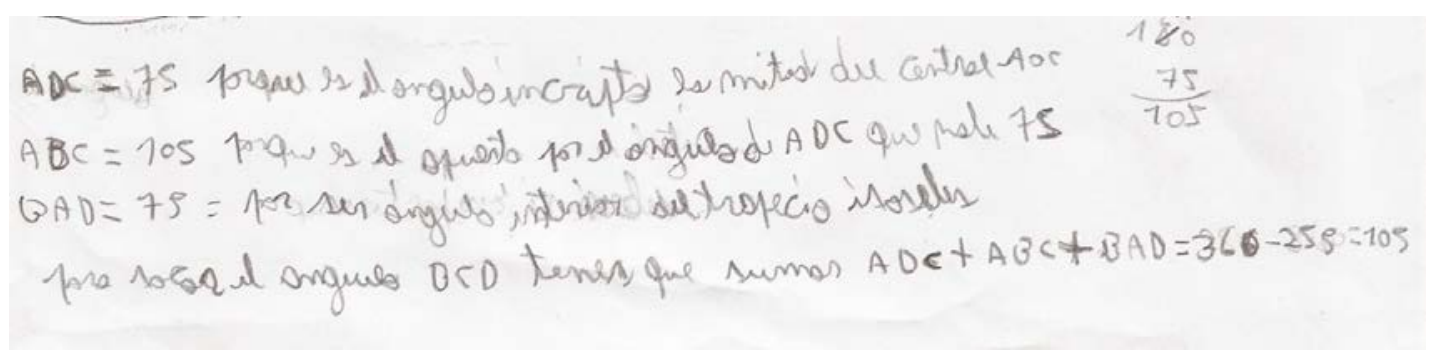

Figura 16. Resolución del Grupo 1 para la situación 11.

El Grupo 1 también explicitó el medio tecnológico, pero a diferencia de los otros grupos, en sus argumentaciones empleó la propiedad de los ángulos interiores de un cuadrilátero para obtener el ángulo $B \hat{C} D$, como se muestra en la figura 17.

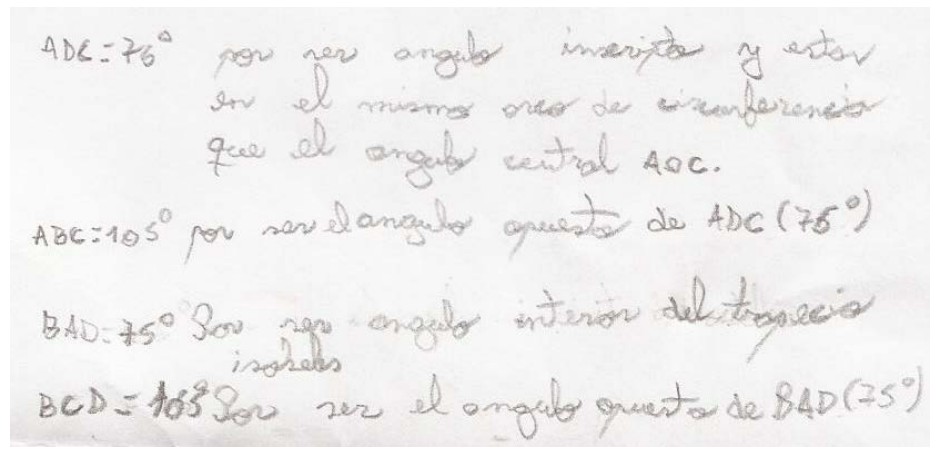

Figura 17. Resolución del Grupo 3 para la situación 11.

En el hacer de la situación destacamos el empleo de la técnica institucionalizada en la situación 9. Los estudiantes obtuvieron las amplitudes de los ángulos independientemente de la experimentación y explicitaron el entorno tecnológico.

\section{COMENTARIOS FINALES}

En este trabajo reportamos el diseño e implementación de una AEI que involucra el estudio de ángulos inscriptos en circunferencia y de cuadriláteros cíclicos.

Esta primera implementación constituyó una prueba piloto de la AEI, en un curso habituado al estudio de la matemática de manera tradicional. Describimos las técnicas y el entorno tecnológico-teórico empleado por los estudiantes en el hacer de la 
AEI, junto al topos del alumno y el profesor. Dentro de la ideología de las AEI, no conseguimos darnos suficiente espacio para poder salir del camino que habíamos planeado recorrer y los encuentros que producir. Pues, no logramos que los estudiantes propongan nuevas cuestiones ante las resoluciones que iban obteniendo. Sin embargo, hemos logrado que se responsabilicen de las tareas elaborando y validando las técnicas propuestas.

La AEI se compuso de actividades de lápiz y papel y con el empleo del software Geogebra $^{\circledR}$. En particular, los estudiantes resolvieron situaciones que permitieron desarrollar razonamientos propios del trabajo geométrico. Los alumnos dedujeron a partir de los datos y utilizaron propiedades y relaciones que no se encontraban explícitas en las situaciones. Las tareas con el empleo de software permitieron que los estudiantes exploren construcciones dinámicas. El modo de arrastre facilitó obtener y estudiar tantos ejemplos como sea necesario en poco tiempo, y que no puede ser obtenido en la enseñanza con lápiz y papel. El uso del programa GeoGebra ${ }^{\circledR}$ permitió la integración de diversas nociones en el estudio de los cuadriláteros, que se proponen estudiar de manera aislada en el diseño curricular. Así mismo, consideramos que la tecnología no fue la determinante en el proceso de aprendizaje, sino la actividad cognitiva de los estudiantes, en la medida en que reflexionaron sobre la actividad matemática que llevaron a cabo. En particular, en algunas producciones de los estudiantes observamos que para justificar las propuestas les era suficiente hacer referencia a la información que brinda el software: el hecho de observar mediciones es suficiente para establecer la verdad de un enunciado. El profesor tuvo que intervenir en estas situaciones, para que los estudiantes justificaran lo que el software les mostraba.

Destacamos las dificultades para desarrollar un dispositivo didáctico con las características de una AEI en la escuela secundaria. Para el grupo de estudiantes con el que se trabajó se percibe que resolver una situación es dar respuesta a la demanda del profesor. Este tipo de respuestas se caracterizan por la ausencia de un entorno tecnológico explícito que justifique la manera de hacer. Probablemente esto se deba a la formación que han recibido los estudiantes durante toda su escolaridad. La noción de AEI es una alternativa incompleta y limitada, pero se trata de una propuesta viable en la 
escuela secundaria y permite comenzar a enfrentar el problema de la monumentalización e instalar algunos elementos de la pedagogía de la investigación.

\section{REFERENCIAS}

ACOSTA, M. (2005). Geometría experimental con Cabri: una nueva praxeología matemática. En Educación Matemática, 17(3), pp. 121-140.

ANDRADE, J. A., NACARATO, A. M. (2004). Tendências didáticopedagógicas no ensino de Geometria: um olhar sobre os trabalhos apresentados nos ENEMs. En Educação Matemática em Revista. 11(17), pp. 61-70.

CHEVALLARD, Y. (1985). La transposition didactique ; du savoir savant au savoir enseigné. La Pensée Sauvage: Paris.

CHEVALLARD, Y. (1999). L'analyse des pratiques enseignantes en théorie anthropologique du didactique. En Recherches en Didactique des Mathématiques. 19/2, pp. 221-266.

CHEVALLARD, Y. (2006). Steps towards a new epistemology in mathematics education. Disponible en: http://yves.chevallard.free.fr

CHEVALLARD, Y. (2007). Passé et présent de la théorie anthropologique du didactique. . Disponible en: http://yves.chevallard.free.fr

CHEVALLARD, Y. (2009a). La notion d'ingénierie didactique, un concept à refonder. Questionnement et éléments de réponse à partir de la TAD. Disponible en: http://yves.chevallard.free.fr

CHEVALLARD, Y. (2009b). La notion de PER: problèmes et avancées. Disponible en: http://yves.chevallard.free.fr

CORICA, A.; MARÍN, E. (2014). Actividad de Estudio e Investigación para la enseñanza de nociones de geometría. En Números, 85, pp.91 -114.

CORRALES, J. (2011). Las construcciones con GeoGebra como medio para resignificar las propiedades de las Figuras. En UNION, 28, pp. 177 189.

DGCyE. (2008). Diseño Curricular Para La Educación Secundaria 2 Año (Sb). Gobierno de la Provincia de Buenos Aires. Matemática. 293-350. 
Disponible

en:

http://abc.gov.ar/lainstitucion/organismos/consejogeneral/disenioscurric ulares/

DGCyE. (2009). Diseño Curricular Para La Educación Secundaria 3 Año (Sb).Gobierno de la Provincia de Buenos Aires. Matemática. 303-376. Disponible en: http://abc.gov.ar/lainstitucion/organismos/consejogeneral/disenioscurric ulares/

GARCÍA, I.; ARRIERO, A, C. (2000). Una experiencia con Cabri: las curvas cónicas. En Suma, 34, pp. 73-80.

GONZÁLEZ, M. J.; LUPIÁÑEZ , J. L. (2001). Formación inicial de profesores de matemáticas de secundaria: actividades basadas en la utilización de software de geometría dinámica. En UNO, 28, pp. 110-125.

HERNÁNDEZ, R.; FERNÁNDEZ, C; BAPTISTA, M. (2010). Metodología de la investigación. México D. F.: Mc Graw Hill.

HOYOS, V. (2006). Funciones complementarias de los artefactos en el aprendizaje de las transformaciones geométricas en la escuela secundaria. En Enseñanza de las ciencias, 24(1), pp. 31-42.

IARANZO N.; FORTUNY J. M. (2009). La influencia conjunta del uso de Geogebra y lápiz y papel en la adquisición de competencia del alumnado. En Enseñanza de las ciencias, 27 (3), pp. 433-445.

ITZCOVICH, H. (2005). Iniciación al estudio didáctico de la Geometría. Buenos Aires: El Zorzal.

LARIOS, V.; GONZALEZ G. N. (2009). Aspectos que influyen en la construcción de la demostración en ambientes de geometría dinámica. En RELIME, 14, pp. 147-160.

MARÍN, E. (2012). Actividad de estudio e investigación para el estudio de ángulos inscriptos en circunferencias. Tesis de Licenciatura en Educación Matemática. Facultad de Ciencias Exactas de la UNCPBA. 
MARRADES, R. Y GUTIERREZ, A. (2000). Proofs produced by secondary school students learning geometry in a dynamic computer environment. Educational Studies in Mathematics, 44, 87-125.

PAULEK, C.; DIAS, M. (2013). Tecnologías de la Información y la Comunicación (TIC): Um Estudo Sobre a Influência do Software GeoGebra na Elaboração das Demonstrações Geométricas. En UNION, 35, pp. $145-160$.

PEREZ, A. (2000). Cabri e Internet. En Suma, 36, pp. 113-115.

PICHEL , J. M. (2000). Requeteoremas: reiventando teoremas en geometría con Cabri II. En Suma, 36, pp. 17-22.

RICHARD, A. (2010). Textos clásicos y geometría dinámica: estudio de un aporte mutuo para el aprendizaje de la geometría. En Enseñanza de las ciencias, 28(1), pp. 95-112. 